Published in final edited form as:

J Magn Reson. 2016 August ; 269: 55-64. doi:10.1016/j.jmr.2016.04.012.

\title{
Experimental and numerical analysis of $B_{1}+$ field and SAR with a new transmit array design for $7 \mathrm{~T}$ breast MRI
}

\author{
Junghwan Kim ${ }^{1,2}$, Narayan Krishnamurthy ${ }^{1}$, Tales Santini ${ }^{1}$, Yujuan Zhao ${ }^{1}$, Tiejun Zhao ${ }^{3}$, \\ Kyongtae Ty Bae ${ }^{1,2}$, and Tamer S. Ibrahim ${ }^{1,2}$ \\ ${ }^{1}$ Department of Bioengineering, University of Pittsburgh, Pittsburgh, PA 15261, USA \\ ${ }^{2}$ Department of Radiology, University of Pittsburgh, Pittsburgh, PA 15213, USA \\ ${ }^{3}$ MR Research Support, Siemens Healthcare, Pittsburgh, PA 15213, USA
}

\section{Graphical Abstract}

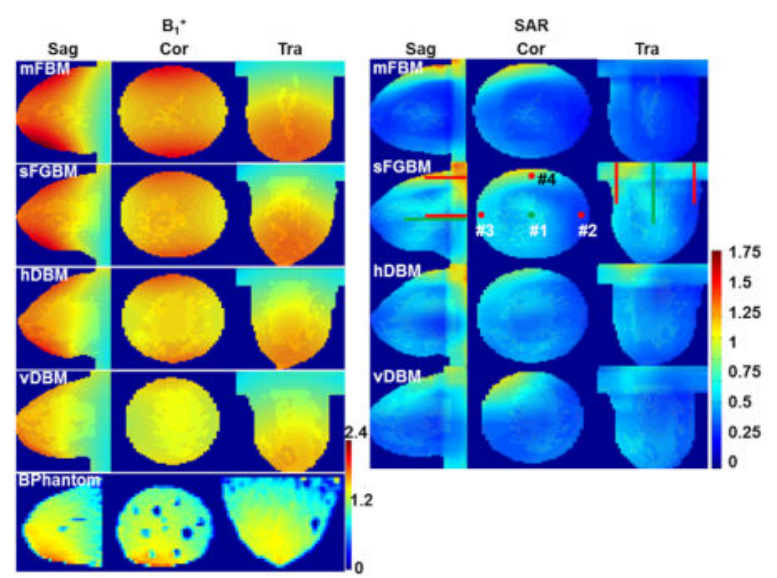

\section{Keywords}

7 Tesla; FDTD; $\mathrm{B}_{1}{ }^{+}$; SAR; Breast; Tx/Rx array; RF coil

\section{Introduction}

For high field MRI, it is critical to design RF coils that produce maximum $\mathrm{B}_{1}{ }^{+}$field (circularly polarized component of the transverse magnetic field responsible for exciting the spins) uniformity within the region of interest (ROI) along with acceptable specific absorption rate (SAR) levels. While taking advantage of increased signal-to-noise ratio (SNR) at 7T, which could be 2-4 times higher than that at 3T, multi transmitter (Tx) arrays

CORRESPONDENCE TO: Tamer S. Ibrahim, Departments of Bioengineering and Radiology, University of Pittsburgh, Pittsburgh, PA 15213, USA, Tel: 412-383-6946, Fax: 412-641-2582, tibrahim@ pitt.edu.

Publisher's Disclaimer: This is a PDF file of an unedited manuscript that has been accepted for publication. As a service to our customers we are providing this early version of the manuscript. The manuscript will undergo copyediting, typesetting, and review of the resulting proof before it is published in its final citable form. Please note that during the production process errors may be discovered which could affect the content, and all legal disclaimers that apply to the journal pertain. 
in conjunction with RF-shimming technique have been widely studied to improve the $\mathrm{B}_{1}{ }^{+}$ field homogeneity and minimize the power deposition in the tissue [1-7]. When the static $\left(\mathrm{B}_{0}\right)$ magnetic field strength increases; reaching $7 \mathrm{~T}$ and beyond, its operating wavelength becomes shorter or comparable to the object being imaged and the interactions between the biological tissue and the electromagnetic fields produced by the RF coil become more complex when compared to that observed at lower magnetic field strengths. At higher fields, constructive/destructive interference can create signal void regions $[8,9]$ with potential higher SARs. Studies have reported that these variations can yield undesired signal changes in breast MRI which can lead to misinterpretation, less reproducibility, inaccuracy of assessing the changes in the ROI and increased SAR [1, 10-14]. It has also been recognized that $\mathrm{B}_{1}{ }^{+}$field inhomogeneity can be improved through a careful increase of the volume of the RF coil [10][15].

RF-shimming can be performed and evaluated in vivo; however, in vivo SAR measurements are not usually considered feasible. Temperature rises due to the RF heating have been studied with phantoms and/or animal models; such studies are however rare in humans due to complexities and safety concerns $[4,6,16-19]$. This has led to extensive use of numerical modeling to predict SAR distributions with anatomically detailed human models for safety evaluations in high field MRI [4-7, 17, 19-24].

In this work, a new RF array system that has multiple excitation sources (8) which can be independently driven (with different magnitude and/or phase) was developed and mathematically analyzed for breast imaging at $7 \mathrm{~T}$. Numerical and experimental studies were performed and quantitatively analyzed to evaluate the RF breast coil. Specifically, we have developed an eight-channel, two sided 2-by-2 cross-pole [25-27] or Tic-Tac-Toe (TTT) 7T $\mathrm{RF}$ array for the breast region. The developed RF array inherently shows high couplings $(\mathrm{S} 13=\sim-3 \mathrm{~dB}$ and $\mathrm{S} 12=-9 \mathrm{~dB})$ between the excitation elements thus the $\mathrm{B}_{1}{ }^{+}$field intensity per supplied (by the system) power is typically lower than the traditional breast RF coils, however, the array exhibits more homogeneous $\mathrm{B}_{1}{ }^{+}$field distributions in the breast tissue. Also, due to high coupling between the coil elements, our design shows less loading dependency than traditional coil designs and therefore does not require tuning, matching or RF shimming from subject to subject.

Additionally while many high field breast MRI studies published promising results [1, 1013, 28-33]; to our knowledge, so far, only one study has evaluated $\mathrm{B}_{1}{ }^{+}$field and peak/ average SAR variations in different breast models and compared to breast phantoms [34]. In our study, numerical analysis was performed using FDTD method in a homogeneous spherical model and in four different breast models. Mean $\mathrm{B}_{1}{ }^{+}$field intensity $(\mu \mathrm{T})$ achieved for 1 Watt of supplied power and CoV (standard deviation/mean) were calculated. Peak and average SAR $\left(\mathrm{W} / \mathrm{kg}\right.$ ) were calculated in order to achieve a $\mathrm{B}_{1}{ }^{+}$field intensity of $2 \mu \mathrm{T}$ in different regions of the breast models. In addition to the different breast model comparisons, $\mathrm{B}_{1}{ }^{+}$field and peak/average SAR were calculated in two additional settings: 1) breast model merged with 3D human female torso, "full body model", to evaluate the influence of the chest regions, and 2) without the RF shield around the breast regions to evaluate the effects of the RF shield [35-37]. Furthermore, experimental $\mathrm{B}_{1}{ }^{+}$field maps were acquired in a homogeneous spherical phantom and in the "Bphantom" which consists of 50\% glandular 
and $50 \%$ adipose tissue with $8-10$ cystic masses typically used for biopsy training (Supertech, IN). The temperature rises due to the RF power absorption were measured using fiber optic probes (Neoptix, Canada) with the same Bphantom.

\section{Materials and Methods}

\section{RF array design}

Transmission line elements (coaxial line with solid squared-shaped inner copper rod and an outer copper strut) were adapted for our two-sided $2 \times 2$ TTT RF coil [37]. The inner copper rod (40 $\mathrm{mm}$ in length) is inserted inside a hollow squared shaped polycarbonate tube which is surrounded with a copper sheet (note, each rod is inserted up to $\sim 40 \mathrm{~mm}$ such that it does not pass through other rods at cross-over joint). The inner copper rods are electrically connected to the surrounding RF copper shield and the outer copper struts are all electrically connected (Figure 1). The array can be tuned and matched by pushing/pulling the inner copper rods inside the outer copper struts which effectively change the lengths of the transmission lines and therefore the associated capacitance and inductance (Figure 1A). We used a total of eight squared shaped coaxial lines measured $25.4 * 25.4 \mathrm{~mm}^{2}$ (on the outside) and $19.0 * 19.0 \mathrm{~mm}^{2}$ (on the inside). The coaxial elements on each side of the four struts were placed in the shape of a tic-tac-toe (Figure 1). On four alternating channels per side, the coaxial center pin of each RF excitation port was connected to the outer strut immediately above it and the ground of the port was connected to the RF shield of the box. Each side was constructed on a $170 \times 170 \mathrm{~mm}^{2}$ acrylic frame (Figure 1A). Two sides were then assembled with a $127 \times 170 \times 170 \mathrm{~mm}^{3}$ copper shield box to decrease the radiation loss and increase RF efficiency [38] (green dotted line in Figure 1B). Approximate lengths of the matching and the tuning rods that need to be inserted in their respective outer struts were calculated using full wave FDTD simulations. After assembly, fine tuning/matching adjustments were then done on the bench. Each side contained four RF excitation ports and all eight excitation ports were tuned and matched to $297.2 \mathrm{MHz}$ and scattering (S) matrix were measured using vector network analyzer (HP, USA). Figure 1C shows the MR images of the Bphantom that was used in this study. The current useable volume of the array (cavity) is $12.7 \times 17.0 \times 17.0 \mathrm{~cm}^{3}$ which is marginally larger than the volume of the breast model/phantom possibly resulting in a lower $\mathrm{B}_{1}{ }^{+}$field intensity for a given RF power supplied by the system. Due to the structure of design (see Figure 1) however, this arrangement has a negative impact on the $\mathrm{B}_{1}{ }^{+}$field homogeneity as the breast phantoms are not positioned inside the array's central volume, "sweet spot", which is positioned at a deeper location towards the magnet table.

\section{FDTD calculations and breast models}

An in-house FDTD model of a 2-sided $2 \times 2$ TTT RF array was developed (Figures 2A-C) where a 3-dimentional (D) geometry of the array was created using MATLAB (Mathworks, MA). A grid matrix composed of $166^{*} 166^{*} 143$ cells with a spatial resolution of (1.587 $\left.\mathrm{mm}^{3}\right)$ and a temporal resolution of $\left(3^{*} 10^{-12} \mathrm{~s}\right)$ was used to meet the Courants stability criterion $[39,40]$. The computational domain was surrounded with 32 layers of perfectly matched layer (PML) as absorbing boundary conditions [41]. 
Four 3D breast models developed at the University of Wisconsin Madison (http:// uwcem.ece.wisc.edu/phantomRepository.html) were used for this study (Figures 3A-D). The breast models used in this study are 1) mostly fat breast model (mFBM, <25\% glandular, $60 * 71 * 50$ grid cells in $\mathrm{x}, \mathrm{y}, \mathrm{z}$-direction), 2) scattered fibroglandular (FG) breast model (sFGBM, 25-50\% glandular, 57*70*61 grid cells), 3) heterogeneously dense breast model (hDBM, 50-75\% glandular, 43*73*49 grid cells) and 4) very dense breast model (vDBM, $>75 \%$ glandular, $40 * 65 * 42$ grid cells) type defined by the American College of Radiology. Each model is comprised of a 3D grid of cubic voxels $\left(0.5 \times 0.5 \times 0.5 \mathrm{~mm}^{3}\right)$ that was later interpolated to match the spatial resolution of the coil geometry. Six different tissues were used and tissues conductivity and dielectric constant were set to that associated with the Larmor frequency of $7 \mathrm{~T}$ ( $297 \mathrm{MHz}$, see Table 1). In addition, a spherical model (diameter $=100 \mathrm{~mm}$, conductivity $=0.46(S / M)$ and Relative Permitivity $=79)$ was also utilized in the FDTD calculations.

To investigate the effect of full body inclusion, we merged sFGBM to virtual family "Ella" model [42]. First, Ella model $\left(530 * 300 * 1680\right.$ grid cells, and $1 \times 1 \times 1 \mathrm{~mm}^{3}$ cubic voxel) was cropped $300 \mathrm{~mm}$ from the top of head to $550 \mathrm{~mm}$ in z-direction which encompasses chest and breast area. Second, cropped model was then interpolated to the spatial resolution of the computational domain $\left(1.587 \mathrm{~mm}^{3}\right)$. Third, since the Ella was developed in supine position where breast tissues were pulled down by gravity, these breast tissues were removed and replaced with sFGBM.

The same sFGBM was also used for simulation with and without RF shield. Total of 40 grid cells from the center of the RF shield ( 20 cells toward head and 20 cells toward foot) were removed. In the FDTD geometrical configuration, the four breast models and the spherical model were centered inside the RF array to mimic the experimental settings (see Figures 2A-B). The electromagnetic fields (both electric and magnetic field) and reflection (Sxx)/ transmission (Sxy) coefficients from eight excitation ports/channels were calculated with the FDTD software using a true transmission line model for excitation [17, 20, 21, 23].

Calculated electromagnetic fields from each port were linearly superimposed with different phases and $\mathrm{B}_{1}{ }^{+}$field distribution and $\mathrm{SAR}$ were obtained. The 1) mean $\mathrm{B}_{1}{ }^{+}$field intensity $(\mu \mathrm{T})$ per $1 \mathrm{~W}$ of RF power supplied by the system and the 2) $\mathrm{CoV}$ of the $\mathrm{B}_{1}{ }^{+}$field were calculated at three different planes and for entire volume for the 4 breast models and the spherical model. In addition, peak SAR (W/kg) was calculated using the following equation and was determined for 10 gram pixel average:

$$
S A R_{(i, j, k)}=\frac{1}{2}\left\{\frac{\sigma_{(i, j, k)}\left(E_{x(i, j, k)}^{2}+E_{y(i, j, k)}^{2}+E_{z(i, j, k)}^{2}\right)}{\rho_{(i, j, k)}}\right\}
$$

Where sigma $(\sigma)$ is the conductivity and rho $(\rho)$ is the density of tissues in the sample. The density of the breast is determined by the tissue composition ratio of fat to connective tissue. $\mathrm{i}, \mathrm{j}$, and $\mathrm{k}$ represent locations in the Cartesian coordinate system. 


\section{Imaging studies and temperature measurements}

All imaging studies were performed on a $7 \mathrm{~T}$ human MR scanner (Siemens Medical System, Erlangen, Germany). We used 1) 4 ports with $90^{\circ}$ phase increments in the clockwise direction (Figure 1A) on one side and 4 ports with $90^{\circ}$ phase increment in counter clockwise direction on the opposing side and 2) equal amplitudes (on all 8 ports) in order to produce an approximate circular polarization (CP) mode. In order to transmit RF power to the eight excitation ports and receive the MR signal, we used 8-way Wilkinson power divider and 8 T/R switches with abovementioned phase set up on the transmit chain.

A 3D B ${ }_{1}^{+}$field map was acquired with modified turbo flash (TFL) sequence [43] where a presaturation module was inserted before each partition to create varied z-magnetization. The presaturation module consists of a rectangular RF pulse (1ms) and followed by a set of trapezoidal gradient crushers applied on all three axes simultaneously. The crusher gradients' duration is $8 \mathrm{~ms}$ with the peak amplitude $=13 \mathrm{mT} / \mathrm{m}$. The design was achieved to minimize the $\mathrm{T} 1$ relaxation effects between the presaturation pulse and excitation pulse. The nominal flip angle (8 steps) for the presaturation pulse was varied from 0 to 160 degree. Sequence parameters were: TR/TE $=2000 / 1.5 \mathrm{~ms}$, voxel Resolution $=3.0 \times 3.0 \times 3.0 \mathrm{~mm}^{3}$, flip angle for excitation pulse $=8$ degrees, data acquisition bandwidth is $1500 \mathrm{~Hz}$ per Pixel. Acquired data was then fitted to the cosine function using MATLAB (Mathworks, MA).

Four thermal fiber optic probes were inserted in the Bphantom in order to measure the temperature rise due to RF power absorption. Locations of the inserted probes were set based on the local hot spots from simulation results shown in Figure 7 (SAR distribution). Three probes were placed $\sim 5-10 \mathrm{~mm}$ from the phantom boundary (30 $\mathrm{mm}$ in depth) and one probe was inserted at the center of the phantom (50 $\mathrm{mm}$ in depth). The RF coil and the Bphantom were placed in the MR scanner for 20 min prior to the experiment. Baseline temperature was measured for $10 \mathrm{~min}$ and the RF pulse was applied with $\mathrm{X}, \mathrm{Y}, \mathrm{Z}$-gradients turned off. $9 \mathrm{~W}$ continuous wave per channel (total of $8 \mathrm{Tx}$ lines) was applied in the Bphantom to induce RF heating. Sequence parameters are; TR/TE $=50 / 1.5 \mathrm{~ms}$ and RF pulse duration $=3 \mathrm{~ms}$. Each scan time was $2 \mathrm{~min}$ long and was sequentially repeated 5 times. Temperature measurements using Neoptix (http://www.neoptix.com/reflex.asp) were captured at the 4 probe locations with the 1 s temporal resolution for a total duration of 40 minutes (10 min prior to the start of the scan, 10 min scan and 20 min after the scan).

\section{Results}

\section{Coupling: simulations and measurements}

$S$ parameters of the TTT array loaded with different breast models were calculated utilizing the FDTD package and compared with experimental measurements (Figure 4). Without retuning/re-matching (numerically as well as experimentally) between different loads, the mean S11 calculated with the 4 different breast models as well as the spherical model was $-20 \mathrm{~dB}$ whereas $\sim-24 \mathrm{~dB}$ was measured with the analyzer (loaded with spherical phantom, and Bphantom as shown Figures 4A-B). The mean transmission coefficient S13 (two excitation ports that are parallel to each other: see Figure 1A) of $\sim-3 \mathrm{~dB}$ was calculated from the simulation which was comparable to the experimental measurements. In addition, mean 
S12 (two orthogonal excitation ports: see Figure 1A) of -9dB was calculated and measured.

\section{$B_{1}{ }^{+}$field distribution and intensity: simulations and measurements}

The mean $\mathrm{B}_{1}{ }^{+}$field intensity (per $1 \mathrm{~W}$ of RF power supplied by the system) calculated from the homogeneous spherical model in sagittal, coronal, transverse planes and entire volume are $3.54,3.47,3.56$, and $3.50(\mu \mathrm{T} / 1 \mathrm{~W})$ whereas measured (using experimentally obtained $\mathrm{B}_{1}{ }^{+}$field map) from the phantom are $3.41,3.33,3.42$, and $3.39(\mu \mathrm{T} / 1 \mathrm{~W})$, respectively. In both the simulations and experiments, high $\mathrm{B}_{1}{ }^{+}$field intensity at the center of the phantom was observed with symmetric $\mathrm{B}_{1}{ }^{+}$field profile which is expected at the $\mathrm{CP}$ mode (Figures $5 \mathrm{~A}-\mathrm{B})$.

Figure 6 shows the $\mathrm{B}_{1}{ }^{+}$field map calculated/measured in the 4 breast models/the Bphantom. The mean $\mathrm{B}_{1}{ }^{+}$field intensity $(\mu \mathrm{T})$ per $1 \mathrm{~W}$ of RF power supplied by the system and $\mathrm{CoV}$ at sagittal, coronal, and transverse plane are summarized in Table 2. Highest normalized mean $\mathrm{B}_{1}{ }^{+}$field intensity was measured in coronal plane while the values in the sagittal and transverse planes were similar for each breast model as well as Bphantom (see Table 2). $\mathrm{B}_{1}{ }^{+}$ field intensity decreases towards the chest wall was clearly visible in sagittal and transverse planes (Figure 6A). mFBM had highest mean $\mathrm{B}_{1}{ }^{+}$field intensity $(\mu \mathrm{T})$ per $1 \mathrm{~W}$ of RF power supplied by the system in entire volume and decreased as the ratio of $\mathrm{FG}$ tissue increase ( $\mathrm{mFBM}-1.61, \mathrm{sFGBM}-1.53, \mathrm{hDBM}-1.51, \mathrm{vDBM}-1.44$, Bphantom -1.36$)$. The lowest $\mathrm{CoV}$ was calculated in vDBM and increased as fat ratio increased (Figure 6C and Table 2). In the coronal plane, the four breast models had relatively low mean $\mathrm{B}_{1}{ }^{+}$field intensity $(\mu \mathrm{T})$ per $1 \mathrm{~W}$ of RF power supplied by the system; however, the standard deviation (SD) was also low therefore high homogeneity (low $\mathrm{CoV}$ ) was achievable, 4-7\%. In the sagittal and transverse planes, larger SD due to the $\mathrm{B}_{1}{ }^{+}$field decay at the chest wall resulted in lower homogeneity, $\mathrm{Cov} \sim 16-26 \%$.

Due to experimental measurement errors in the BPhantom (inside the cyst masses) highest $\mathrm{CoV}$ was measured in the Bphantom in all three planes, especially in coronal plane (Figure 6B). However, the volume average measurements showed comparable results which varied between $18 \%$ and $26 \%$ (Figure $6 \mathrm{C}$ ). In addition, when the chest wall was excluded in all four breast models (see white dotted line in Figure 6A), the $\mathrm{CoV}$ decreased significantly $(\mathrm{mFBM}-13 \%, \mathrm{sFGBM}-12 \%, \mathrm{hDBM}-11 \%$ and $\mathrm{vDBM}-9 \%)$.

\section{SAR simulations and temperature measurements}

The SAR (per a mean $\mathrm{B}_{1}{ }^{+}$field intensity of $2 \mu \mathrm{T}$ ) distribution associated with the calculated $\mathrm{B}_{1}{ }^{+}$field is shown in Figure 7. Peak/average SAR (W/kg per 10g of tissue) calculated for all breast models are summarized in Table 2. The highest peak and average SAR were calculated in SFGBM. Lowest peak SAR was calculated in vDBM whereas lowest average SAR was calculated in $\mathrm{mFBM}$. The peak/average SAR ratio was 3.28 in $\mathrm{mFBM}, 2.73$ in sFGBM, 2.51 in hDBM and 2.40 in vDBM; the ratio decreased as the composition of FG tissue increases. Regardless of the model, the peak SAR was observed relatively close to the skin and chest wall where the conductivity was high. The SAR maps calculated in the 
mFBM and vDBM model show rapid decay toward the center of the breast model whereas sFGBM and hDBM show relatively uniform distribution (Figure 7).

The temperature rise due to RF heating was measured in the Bphantom. Figure 7 shows probe locations and temperature changes during the RF heating created by the RF excitation described in the Methods Section. Ch1 was placed at the center of the Bphantom. Ch2 and Ch3 were placed to validate the asymmetry of the SAR calculated in the right and left side of the models. Ch4 was placed in the region where peak SAR was calculated (Figure 7). The baseline temperature at the four probes was $\sim 18.5 \mathrm{C}^{\circ}$ and during the total of five RF heating experiments (total $10 \mathrm{~min}$ ), temperature rises were $0.31 \mathrm{C}^{\circ}$ in $\mathrm{Ch} 1,0.12 \mathrm{C}^{\circ}$ in $\mathrm{Ch} 2,0.30 \mathrm{C}^{\circ}$ in $\mathrm{Ch} 3$ and $0.52 \mathrm{C}^{\circ}$ in $\mathrm{Ch} 4$. As shown in Figure $7 \mathrm{C}, \mathrm{Ch} 1$ temperature measurement shows a short linear increase between time $=620-650 \mathrm{Sec}$; steady state was then observed for 120 Sec between time $=650-770 \mathrm{Sec}$. Additional $\sim 0.2 \mathrm{C}^{\circ}$ increase was observed until the end of the measurement $(\sim 2000 \mathrm{Sec})$. Lowest temperature rise was measured in $\mathrm{Ch} 2 . \mathrm{Ch} 3$ temperature rise started to occur around $\sim 840 \mathrm{Sec}$ and continuously increased during the experiment (Figure 7B). Ch4 temperature increased linearly during the first scan (600-720 Sec) and steady state was observed ( $720-840 \mathrm{sec})$ during the second scan. Ch4 temperature reached steady state followed by a short increase during the third scan (Figure 7B). Ch4 temperature had the largest temperature rise which was 4.3 times higher than $\mathrm{Ch} 2$ temperature. In addition, SAR was calculated for the four breast models at $\mathrm{Ch} 2$ and $\mathrm{Ch} 4$ probe locations. The SAR calculated at Ch4 location was $~ 3.5-5$ times higher than that calculated at $\mathrm{Ch} 2$ location. The measured temperature rises as well as calculated SAR values at $\mathrm{Ch} 1$ and $\mathrm{Ch} 3$ locations were comparable.

\section{Inclusion of torso}

Mean $\mathrm{B}_{1}{ }^{+}$field intensity $(\mu \mathrm{T})$ per $1 \mathrm{~W}$ of RF power supplied by the system calculated in the breast tissue regions with the full body model included was 1.26. Although mean $\mathrm{B}_{1}{ }^{+}$field intensity $(\mu \mathrm{T})$ per $1 \mathrm{~W}$ of RF power supplied by the system decreased by $\sim 20 \%$ when compared to sFGBM alone, $\mathrm{CoV}$ calculated in the breast regions with the full body model in $\mathrm{Sag} / \mathrm{Cor} / \mathrm{Tra}$ directions were $0.2 / 0.09 / 0.22$, respectively which are comparable to that calculated in the SFGBM (see Table 2) without the full body model. While the average SAR with attached full body model did not change (using the same regions in both calculations), the peak SAR increased by $\sim 30 \%$.

\section{RF shielding effect}

Mean $\mathrm{B}_{1}{ }^{+}$field intensity $(\mu \mathrm{T})$ per $1 \mathrm{~W}$ of RF power supplied by the system of 0.82 was calculated in the identical sFGBM without RF shield which was $\sim 45 \%$ lower than that calculated in the sFGBM with RF shield. Calculated CoV without RF shield in Sag/Cor/Tra and entire volume were $0.30 / 0.14 / 0.34$ and $0.30 . \mathrm{B}_{1}{ }^{+}$field inhomogeneity increased significantly without RF shield when compared to that calculated in the SFGBM with RF shield. In addition, Peak/average SAR was $\sim 2$ times higher than that calculated in SFGBM with RF shield (3.57/1.34 VS 1.75/0.64 W/kg per 10g, see Table 3). 


\section{Discussions and Conclusion}

The developed transmit breast array inherently exhibits high coupling ( $\mathrm{S} 13=\sim-3 \mathrm{~dB}$ and $\mathrm{S} 12=-9 \mathrm{~dB})$ between the excitation elements thus the average mean $\mathrm{B}_{1}{ }^{+}$field intensity $(\mu \mathrm{T})$ per $1 \mathrm{~W}$ of RF power supplied by the system is typically lower than traditional breast coils $[10,31]$. Although, the average $\mathrm{B}_{1}{ }^{+}$field intensity per SAR is not lower than other traditional RF breast array designs, the proposed design exhibits more homogeneous $\mathrm{B}_{1}{ }^{+}$ field distributions in the breast tissue (despite the fact that breast is not positioned at the coil's central volume or sweet spot). Also, due to high coupling between the coil elements, our design shows less load dependency and therefore tuning, matching, and RF shimming are not required per subject basis whether operating in the parallel transmission (pTx) or single transmission (sTx) modes in order to optimize the performance of the proposed array. By adjusting the rod lengths associated with the excitation elements, one can consistently achieve a matching of $\sim-24 \mathrm{~dB}$ whereas changing the adjacent rods enables the tuning (See figure 1A). This design of the RF array does not require lumped elements for tuning and/or matching. In addition, resonant frequency of the developed array is mainly determined by the geometry inductance, thus the dimension of the array should be carefully chosen based on full wave numerical simulations.

Due to the rigorous modeling of the coil including transmission line excitation, figures $4 \mathrm{~A}-$ B demonstrate very good agreement between experiment and simulations where we accurately calculate the coupling parameters which are high, and therefore well-defined in our array. Such agreement is critical in terms of assessing the electromagnetic fields during the RF coil development and evaluation. Phantom studies showed very good agreement in terms of the $\mathrm{B}_{1}{ }^{+}$field distribution as well as intensity. $\mathrm{B}_{1}{ }^{+}$field distributions/intensities in four breast models and Bphantom were highly consistent and were compared to that of other published high field numerical studies in the breast tissues [10, 31, 34]. Brown et al. (Ref. \#10) showed more than 75\% homogeneity within the breast tissue and McDougall et al. (Ref, \#31) demonstrated approximately $80 \%$ homogeneity in the breast tissues. Although $\mathrm{B}_{1}{ }^{+}$field intensity per supplied (by the system) power was lower than other traditional breast RF coil design by $\sim 20-25 \%, \mathrm{~B}_{1}{ }^{+}$field homogeneity of $\sim 90 \%$ (utilizing the standard quadrature set-up and without utilizing any subject specific RF shimming) in the breast tissue was achievable with our design (note that the $\mathrm{B}_{1}{ }^{+}$field distributions in our study are presented in a linear scale whereas it was noted as dB scale in Ref, \#31). Note that we achieved quadrature excitation (approximate $\mathrm{CP}$ mode) with $90^{\circ}$ phase increments on each side of the array (with opposite rotation) and equal amplitudes in the sTx or combined mode. Furthermore if needed, the developed Tx array can be used in a pTx mode where amplitudes and phases can be optimized in order to further improve the $\mathrm{B}_{1}{ }^{+}$field homogeneity and/or SAR.

Changes of the ratio (FG/fat tissue) affected the mean $\mathrm{B}_{1}{ }^{+}$field intensity ( $\left.\mu \mathrm{T}\right)$ per $1 \mathrm{~W}$ of RF power supplied by the system and $\mathrm{CoV}$ in the breast models. Mean $\mathrm{B}_{1}{ }^{+}$field intensity $(\mu \mathrm{T})$ per $1 \mathrm{~W}$ of RF power supplied by the system decreases as the ratio of FG tissue increases. This effect can be explained as the dielectric constant and conductivity of fat is lower than the FG tissue thus the RF penetration depth is higher in the mFBM than in vDBM. In Contrast, lowest $\mathrm{CoV}$ (best homogeneity) was calculated in the more conductive vDBM due 
to having less predominant dielectric effects. Also, the peak/average SAR ratio varied between 3.28 for lowest FG/Fat model, to 2.40 for highest FG/fat model.

Under the influence of the torso where dielectric constant and conductivity of the tissues were relatively higher than the breast and fat tissues, mean $\mathrm{B}_{1}{ }^{+}$field intensity $(\mu \mathrm{T})$ per $1 \mathrm{~W}$ of RF power supplied by the system decreased by $\sim 20 \%$ in the breast region with a full body model attached. On the other hand, $\mathrm{CoV}$ in the breast region and average SAR with a full body model attached were comparable to that measured in SFGBM alone. Due to the high coupling between the excitation ports, the $\mathrm{B}_{1}{ }^{+}$field distributions are dominated by the coil structure rather than the sample. In addition, when mean $\mathrm{B}_{1}{ }^{+}$field intensity $(\mu \mathrm{T})$ and peak/ average SAR were calculated in the absence of the RF shield, a significant decrease of $\mathrm{B}_{1}{ }^{+}$ field and a significant increase in the SAR were calculated. While there was a limitation of using 4 probes to accurately represent the temperature distribution in the Bphantom, temperature measurements near the edges and near the center support the SAR distributions calculated in the breast models.

When designing new RF coil at high field MRI, accurate numerical prediction of $\mathrm{B}_{1}{ }^{+}$field and SAR is critical to ensure the safety of the patient and to protect the RF hardware. In this study, we evaluated the performance of a new 8-ch RF array developed for breast MR imaging at 7T. While less efficient in terms of mean $\mathrm{B}_{1}{ }^{+}$field intensity per supplied RF power due to RF coupling, this RF array shows homogeneous $\mathrm{B}_{1}{ }^{+}$field distributions in the breast region. Very good agreement between the simulation results and experimental measurements were observed. Future will work will include constructing an identical arrangement for bilateral imaging and combining the proposed Tx array with an Rx-only insert in order to increase SNR in the breast region.

\section{References}

1. Azlan CA, et al. B1 transmission-field inhomogeneity and enhancement ratio errors in dynamic contrast-enhanced MRI (DCE-MRI) of the breast at 3T. Journal of Magnetic Resonance Imaging. 2010; 31(1):234-239. [PubMed: 20027594]

2. de Moortele V, et al. B1 destructive interferences and spatial phase patterns at $7 \mathrm{~T}$ with a head transceiver array coil. Magnetic resonance in medicine. 2005; 54(6):1503-1518. [PubMed: 16270333]

3. Diehl, D., et al. B1 Homogenization at 3 T MRI using a 16 rung transmit array. Proceedings of the 13th Annual Meeting of ISMRM; Miami Beach, FL, USA. 2005.

4. Ibrahim T, et al. Analysis of B1 field profiles and SAR values for multi-strut transverse electromagnetic RF coils in high field MRI applications. Physics in medicine and biology. 2001; 46(10):2545. [PubMed: 11686274]

5. van den Bergen B, et al. 7 T body MRI: B1 shimming with simultaneous SAR reduction. Physics in medicine and biology. 2007; 52(17):5429. [PubMed: 17762096]

6. Cloos, M.; Bonmassar, G. Towards direct B1 based local SAR estimation. Proceedings of the 17th Annual Meeting of ISMRM; Honolulu, Hawaii, USA. 2009.

7. van den Bergen $B$, et al. SAR and power implications of different RF shimming strategies in the pelvis for 7T MRI. Journal of Magnetic Resonance Imaging. 2009; 30(1):194-202. [PubMed: 19557737]

8. Ibrahim TS, Hue YK, Tang L. Understanding and manipulating the RF fields at high field MRI. NMR in Biomedicine. 2009; 22(9):927-936. [PubMed: 19621335] 
9. Ibrahim TS, et al. In-depth study of the electromagnetics of ultrahigh-field MRI. Nmr in Biomedicine. 2007; 20(1):58-68. [PubMed: 17006885]

10. Brown R, et al. Breast MRI at 7 Tesla with a bilateral coil and robust fat suppression. Journal of Magnetic Resonance Imaging. 2014; 39(3):540-549. [PubMed: 24123517]

11. By S, et al. A 16-Channel Receive, Forced Current Excitation Dual-Transmit Coil for Breast Imaging at 7T. PloS one. 2014; 9(11):e113969. [PubMed: 25420018]

12. Harvey JA, et al. Breast MR Imaging Artifacts: How to Recognize and Fix Them 1. Radiographics. 2007; 27(suppl_1):S131-S145. [PubMed: 18180223]

13. Kuhl CK, et al. Effect of B1 inhomogeneity on breast MR imaging at 3.0 T. Radiology. 2007; 244(3):929-930. [PubMed: 17709843]

14. Zastrow, E., et al. Department of Electrical and Computer Engineering University of WisconsinMadison [Online]. 2008. Database of 3D grid-based numerical breast phantoms for use in computational electromagnetics simulations.

15. Vaughan JT, et al. Whole-body imaging at 7T: Preliminary results. Magnetic Resonance in Medicine. 2009; 61(1):244-248. [PubMed: 19097214]

16. Chen J, Feng Z, Jin J-M. Numerical simulation of SAR and B/sub 1/-field inhomogeneity of shielded RF coils loaded with the human head. Biomedical Engineering, IEEE Transactions on. 1998; 45(5):650-659.

17. Dimbylow P. FDTD calculations of the whole-body averaged SAR in an anatomically realistic voxel model of the human body from $1 \mathrm{MHz}$ to $1 \mathrm{GHz}$. Physics in medicine and biology. 1997; 42(3):479. [PubMed: 9080530]

18. Oh S, et al. Experimental and numerical assessment of MRI-induced temperature change and SAR distributions in phantoms and in vivo. Magnetic Resonance in Medicine. 2010; 63(1):218-223. [PubMed: 19785018]

19. Wang Z, et al. SAR and temperature: simulations and comparison to regulatory limits for MRI. Journal of Magnetic Resonance Imaging. 2007; 26(2):437-441. [PubMed: 17654736]

20. Collins CM, Smith MB. Spatial resolution of numerical models of man and calculated specific absorption rate using the FDTD method: a study at $64 \mathrm{MHz}$ in a magnetic resonance imaging coil. Journal of Magnetic Resonance Imaging. 2003; 18(3):383-388. [PubMed: 12938138]

21. Ibrahim TS, Tang L. Insight into RF power requirements and B1 field homogeneity for human MRI via rigorous FDTD approach. Journal of Magnetic Resonance Imaging. 2007; 25(6):12351247. [PubMed: 17520721]

22. Liu F, et al. Numerical modeling of $11.1 \mathrm{~T}$ MRI of a human head using a MoM/FDTD method. Concepts in Magnetic Resonance Part B: Magnetic Resonance Engineering. 2005; 24(1):28-38.

23. Wang H, et al. An MRI-dedicated parallel FDTD scheme. Concepts in Magnetic Resonance Part B: Magnetic Resonance Engineering. 2007; 31(3):147-161.

24. Zhao H, Crozier S, Liu F. Finite difference time domain (FDTD) method for modeling the effect of switched gradients on the human body in MRI. Magnetic resonance in medicine. 2002; 48(6): 1037-1042. [PubMed: 12465114]

25. Ibrahim, TS.; YZ; Zhao, T.; Krishnamurthy, N.; Wood, S.; Raval, S.; Kim, H. 20-to-8 Channel Tx Array with 32-channel Adjustable Receive-Only Insert for 7T Head Imaging. The 21st International Society of Magnetic Resonance in Medicine Annual Meeting; 2013; Salt Lake City, Utah.

26. Zhao, Y.; NK; Wood, S.; Zhao, T.; Raval, SB.; Ibrahim, TS. 3D Eigenmodes Optimizations for 3D Imaging at 7T. The 23nd International Society of Magnetic Resonance in Medicine Annual Meeting; 2015; Toronto, Canada.

27. Zhao, Y.; TZ; Krishnamurthy, N.; Ibrahim, TS. On the E-Field Construction/Deconstruction and B1+ Efficiency/Homogeneity with Transmit Array Eigen Modes. The 22nd International Society of Magnetic Resonance in Medicine Annual Meeting; 2014; Milan, Italy.

28. Cui, J., et al. A switched-mode breast coil for 7 tesla MRI using forced-current excitation. 2015.

29. Klomp D, et al. High resolution MR imaging and spectroscopy of the human breast at 7T using a focused field RF coil setup. Proc Intl Soc Mag Reson Med. 2010

30. Korteweg MA, et al. Feasibility of 7 Tesla Breast Magnetic Resonance Imaging Determination of Intrinsic Sensitivity and High-Resolution Magnetic Resonance Imaging, Diffusion-Weighted 
Imaging, and 1H-Magnetic Resonance Spectroscopy of Breast Cancer Patients Receiving Neoadjuvant Therapy. Investigative Radiology. 2011; 46(6):370-376. [PubMed: 21317792]

31. McDougall MP, et al. Quadrature transmit coil for breast imaging at 7 tesla using forced current excitation for improved homogeneity. Journal of Magnetic Resonance Imaging. 2014; 40(5):11651173. [PubMed: 24459091]

32. Stehouwer B, et al. 101 Feasibility of Contrast-enhanced and High-resolution 7 Tesla MRI in Patients with Suspicious Breast Lesions. European Journal of Cancer. 2012; 48:S70.

33. Zheng T, et al. A 7-Tesla high density Tx/Rx mammography coil. Proc Intl Soc Mag Reson Med. 2011

34. van der Velden TA, et al. Radiofrequency configuration to facilitate bilateral breast 31P MR spectroscopic imaging and high-resolution MRI at 7 Tesla. Magnetic Resonance in Medicine. 2015; 74(6):1803-1810. [PubMed: 25521345]

35. Avdievich NI, Krymov VN, Hetherington HP. Modified perturbation method for transverse electromagnetic (TEM) coil tuning and evaluation. Magnetic resonance in medicine. 2003; 50(1): 13-18. [PubMed: 12815673]

36. Ibrahim TS, et al. Effect of RF coil excitation on field inhomogeneity at ultra high fields: a field optimized TEM resonator. Magnetic Resonance Imaging. 2001; 19(10):1339-1347. [PubMed: 11804762]

37. Roschmann, PKH. High-frequency coil system for a magnetic resonance imaging apparatus. Google Patents; 1988.

38. MD H. Radiative losses of a birdcage resonator. Magn Reson Med. 1993; 29:713-16. [PubMed: 8505911]

39. Taflove, A.; HS. Computational Electrodynamics: The Finite-Difference Time-Domain Method. 2005.

40. Hand J. Modelling the interaction of electromagnetic fields (10 MHz-10 GHz) with the human body: methods and applications. Physics in medicine and biology. 2008; 53(16):R243. [PubMed: 18653928]

41. Berenger J-P. Three-dimensional perfectly matched layer for the absorption of electromagnetic waves. Journal of computational physics. 1996; 127(2):363-379.

42. Andreas C, et al. The Virtual Family-development of surface-based anatomical models of two adults and two children for dosimetric simulations. Physics in Medicine and Biology. 2010; 55(2):N23. [PubMed: 20019402]

43. Fautz $\mathrm{H}$, et al. Extending the sensitivity range for transmit array B1 mapping using relative B1 maps. Proc Intl Soc Mag Reson Med. 2012; 20:3363. 


\section{Highlights}

- $\quad$ Simulated S-parameters of developed RF array matched well with the experimental measurements

- $\quad$ Mean $\mathrm{B}_{1}{ }^{+}$decreased as the ratio of fibroglandular (FG) tissue increase

- Highest $\mathrm{B}_{1}{ }^{+}$homogeneity was calculated in more conductive breast model

- $\quad$ Average/peak SAR maps were calculated in the breast models and full body model

- $\quad$ The temperature measurements supported the SAR distributions calculated in the breast models 

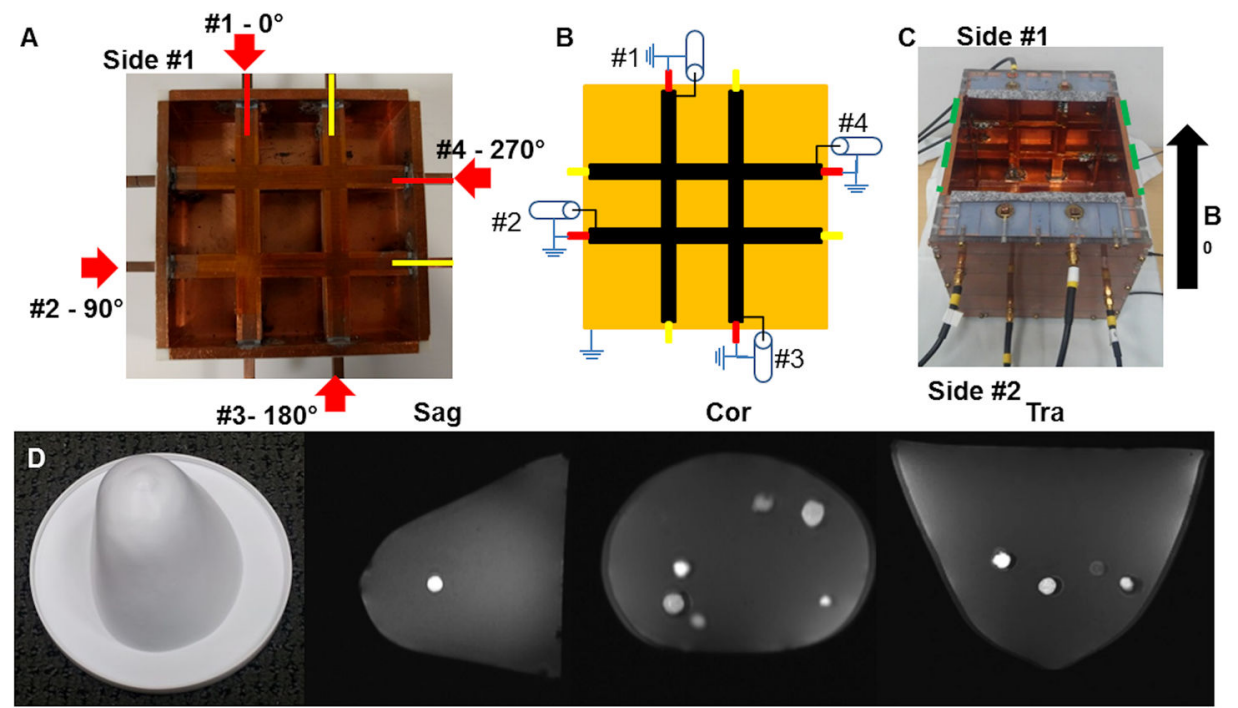

Figure 1.

Constructed TTT Tx array, schematic diagram of one side of the array and breast phantom (AD). (A) One side of TTT RF array showing four excitation ports. Phases $\left(90^{\circ}\right.$ increment) applied in the side \#1 were in a clockwise rotation whereas the same phases were applied in counterclockwise rotation in side \#2. Red arrows represent four excitation ports that can be matched by pulling/pushing the solid copper rods (red bar represent matching rods and yellow bar represent tuning rods). (B) Schematic of one side of Tx coil showing electrical connection between each ports and RF shield. Red/yellow bars represent the inner matching and tuning copper rods connected to the RF ground and orange box represents RF shield. Black lines and bars represent the electrical connections to the four excitation center pins. (C) Top view of assembled RF coil. RF shield box (green dotted line) was attached to the two sides. (D) Picture and MR images of the BPhanom in three different planes. Dimensions of the BPhantom are $120 * 100 * 90 \mathrm{~mm}^{3}$ and the volume is $500 \mathrm{cc}$. 


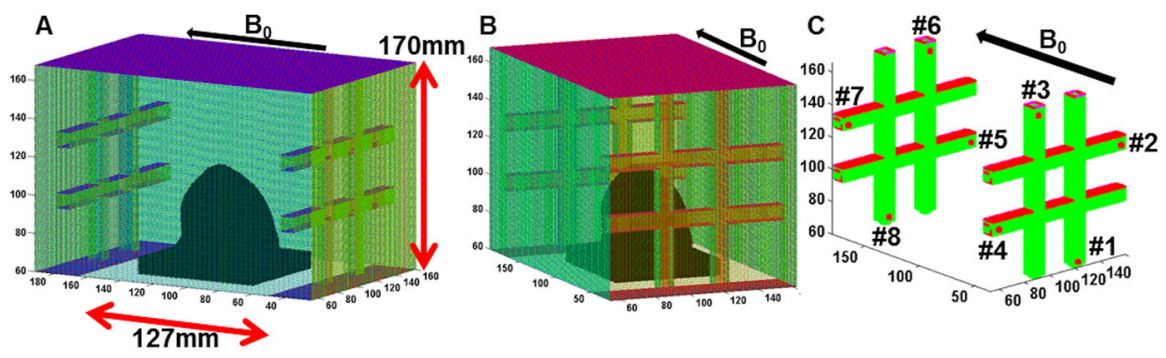

Figure 2.

FDTD model of TTT Tx array with 3D breast model co-centered at the bottom of the coil (A and B). Total grid of $162 * 162 * 168$ cells was used in the computational domain. Sagittal (A) and transverse (B) plane views of the RF coil and the breast model are shown. Two sides of $170 \times 170 \mathrm{~mm}^{2} \mathrm{RF}$ array were $127 \mathrm{~mm}$ apart and RF shield box dimensions were $127 \times$ $170 \mathrm{~mm}^{2}$. (C) Eight excitation ports (no RF shield) are shown. Red dot represents the excitation source points at each port 


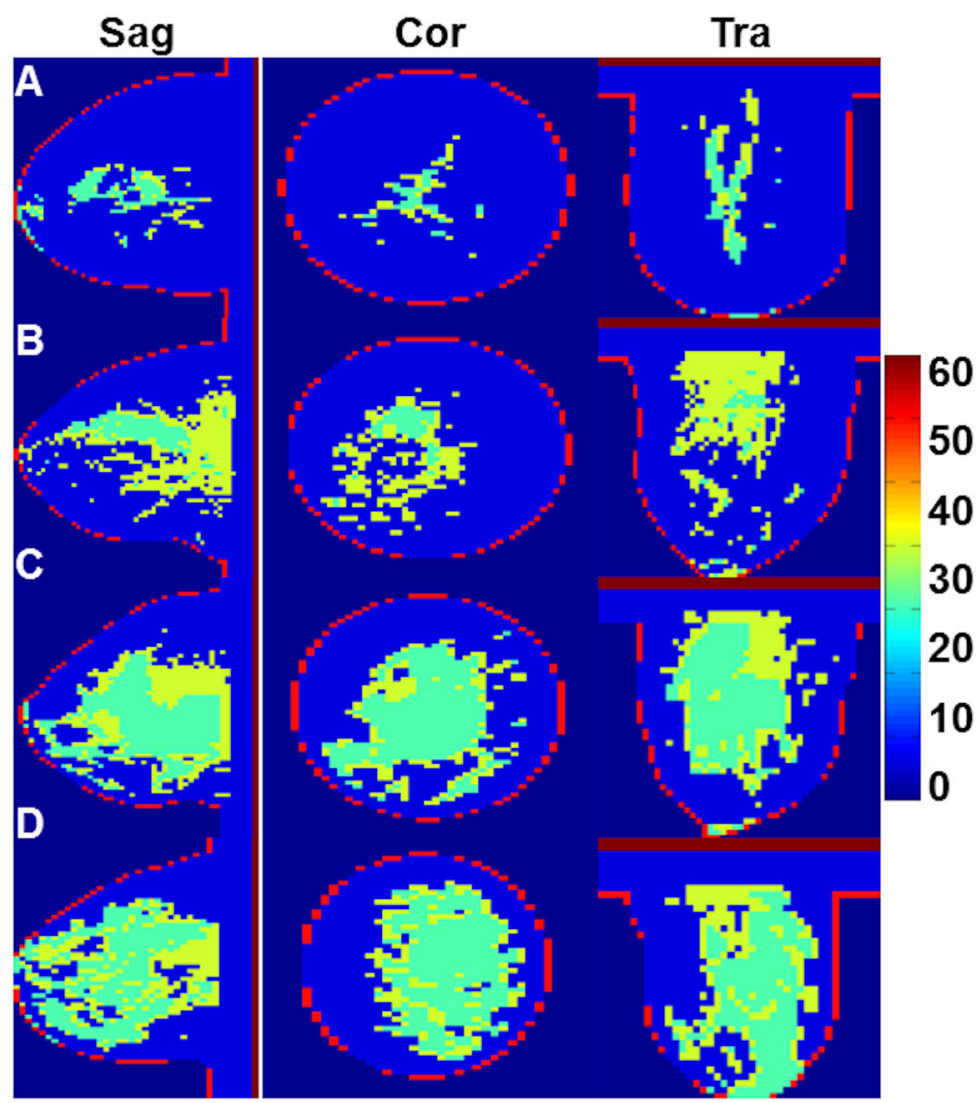

Figure 3.

Permittivity map of four 3D anatomically detailed breast models used in FDTD calculations. (A) mFBM: <25\% glandular (B) sFGBM: 25-50\% glandular (C) hDBM: 50-75\% glandular and (D) vDBM: $>75 \%$ glandular. The phantom models consisted of six tissues with air surrounding. The colorbar represents the relative permittivity of the tissues in the model. 

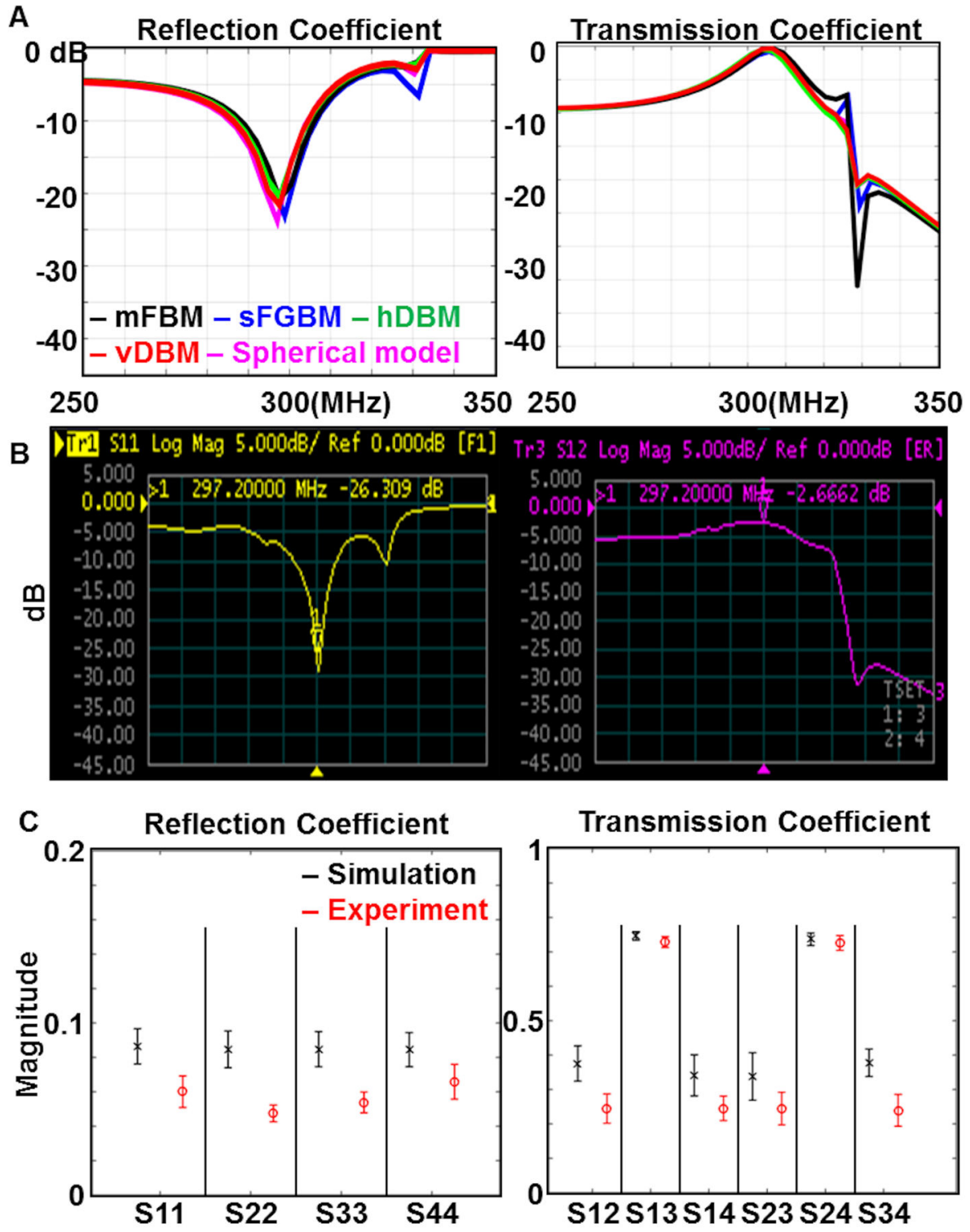

Figure 4.

$\mathrm{S}$-matrix calculated from four breast models and spherical model and measured from the Bphantom and spherical phantom. (A) FDTD calculated S11 and S13 comparison between four different breast models and the spherical model. (B) Measured S11 and S13 of the spherical phantom utilizing vector network analyzer (showing very good agreement with (A)). Note that, the high coupling $(\sim-3 \mathrm{~dB})$ at S13 is intrinsic characteristic of the TTT RF coil. (C) The mean transmission/reflection coefficient comparison between the simulations and experiments. The simulations were obtained with the four breast models and the spherical model whereas the experiments were obtained with the spherical phantom and the Bphantom. 

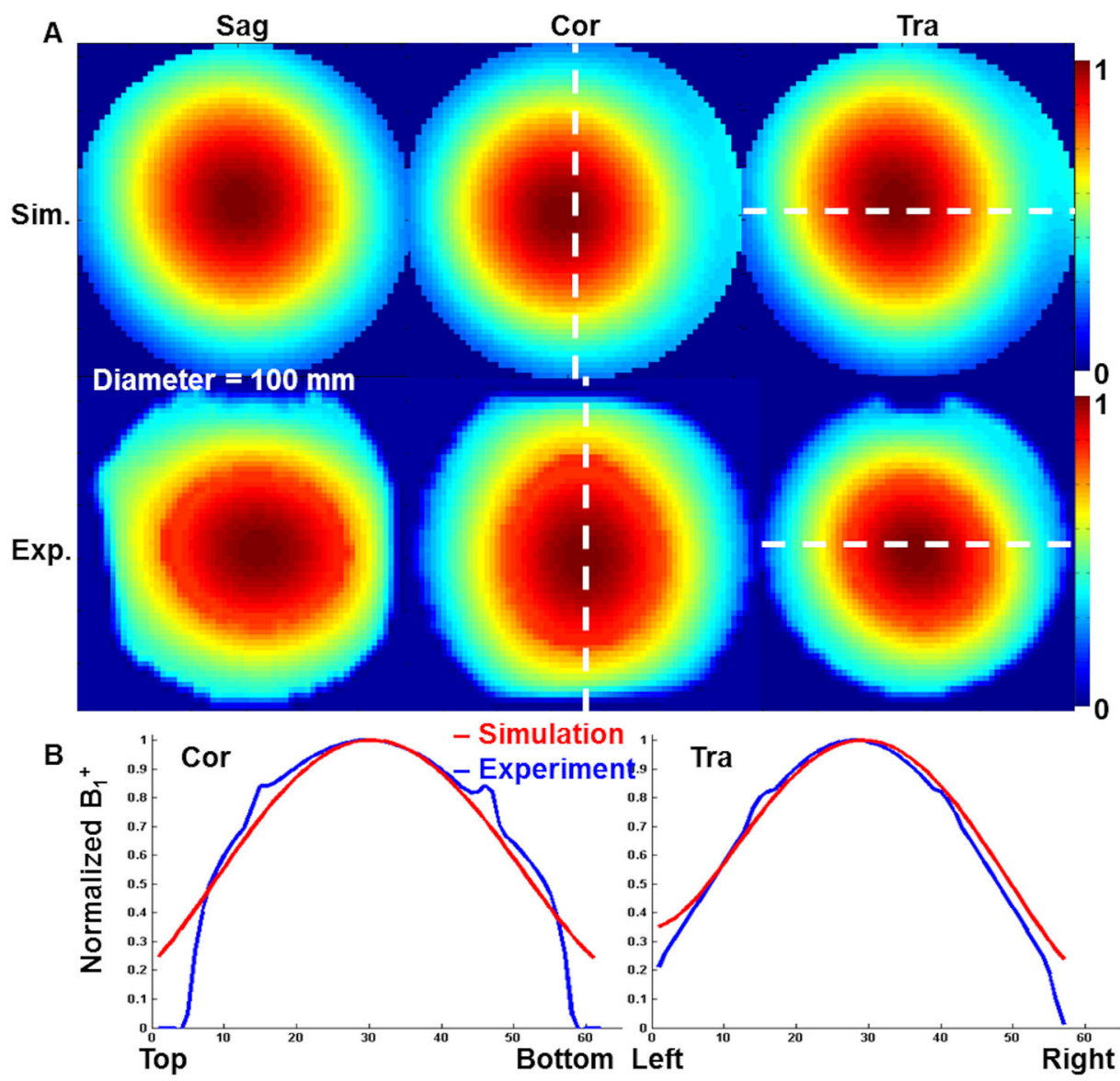

Figure 5.

(A) Simulated/measured $\mathrm{B}_{1}{ }^{+}$distribution from the homogeneous spherical phantom at three different planes $\left(\mathrm{B}_{1}{ }^{+}\right.$was normalized with the local maximum $\mathrm{B}_{1}{ }^{+}$intensity). (B) Signal profile comparison (white dotted line in $(\mathrm{A})$ ) in the coronal and transverse planes; $\mathrm{x}$ axis represents pixel points. Diameter of the phantom/model is $100 \mathrm{~mm}$. 


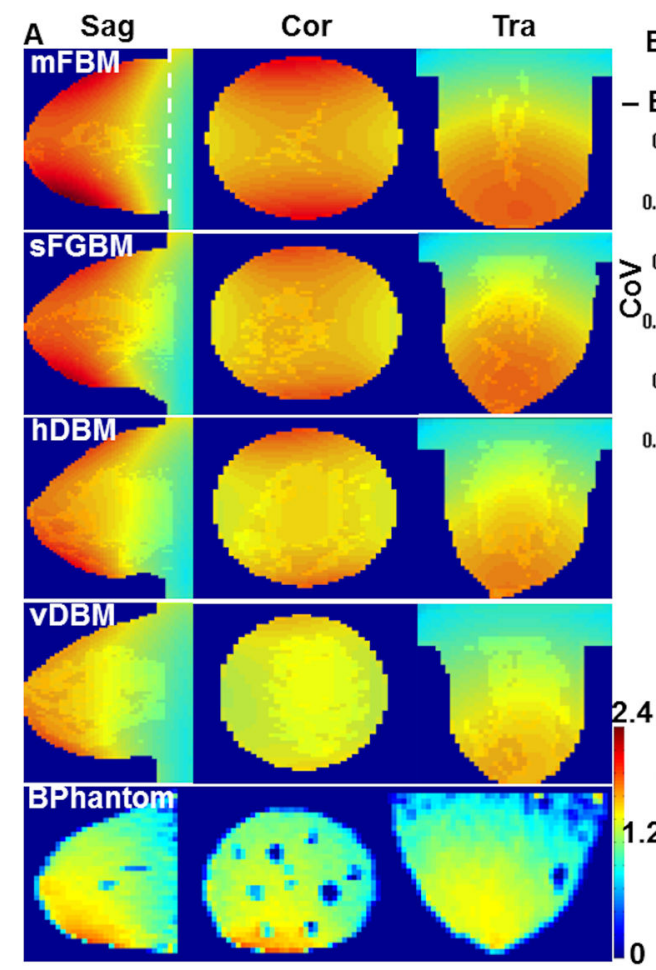
B

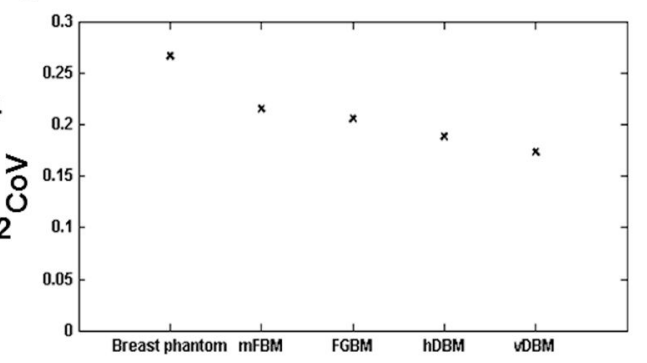

Figure 6.

(A) Mean $\mathrm{B}_{1}{ }^{+}$field intensity $(\mu \mathrm{T})$ per $1 \mathrm{~W}$ of $\mathrm{RF}$ power supplied by the system calculated in the four breast models (simulations) and measured experimentally in the Bphantom. Note, white dotted line is the border of the chest wall used for $\mathrm{CoV}$ calculation labeled "without the chest wall". $\mathrm{B}_{1}{ }^{+}$map was normalized to the maximum $\mathrm{B}_{1}{ }^{+}$measured in the model (2.38 $\mu \mathrm{T}$ in $\mathrm{mFBM}$ ). (B) Mean $\mathrm{CoV}$ at three different planes. (C) CoV over the entire volume. 

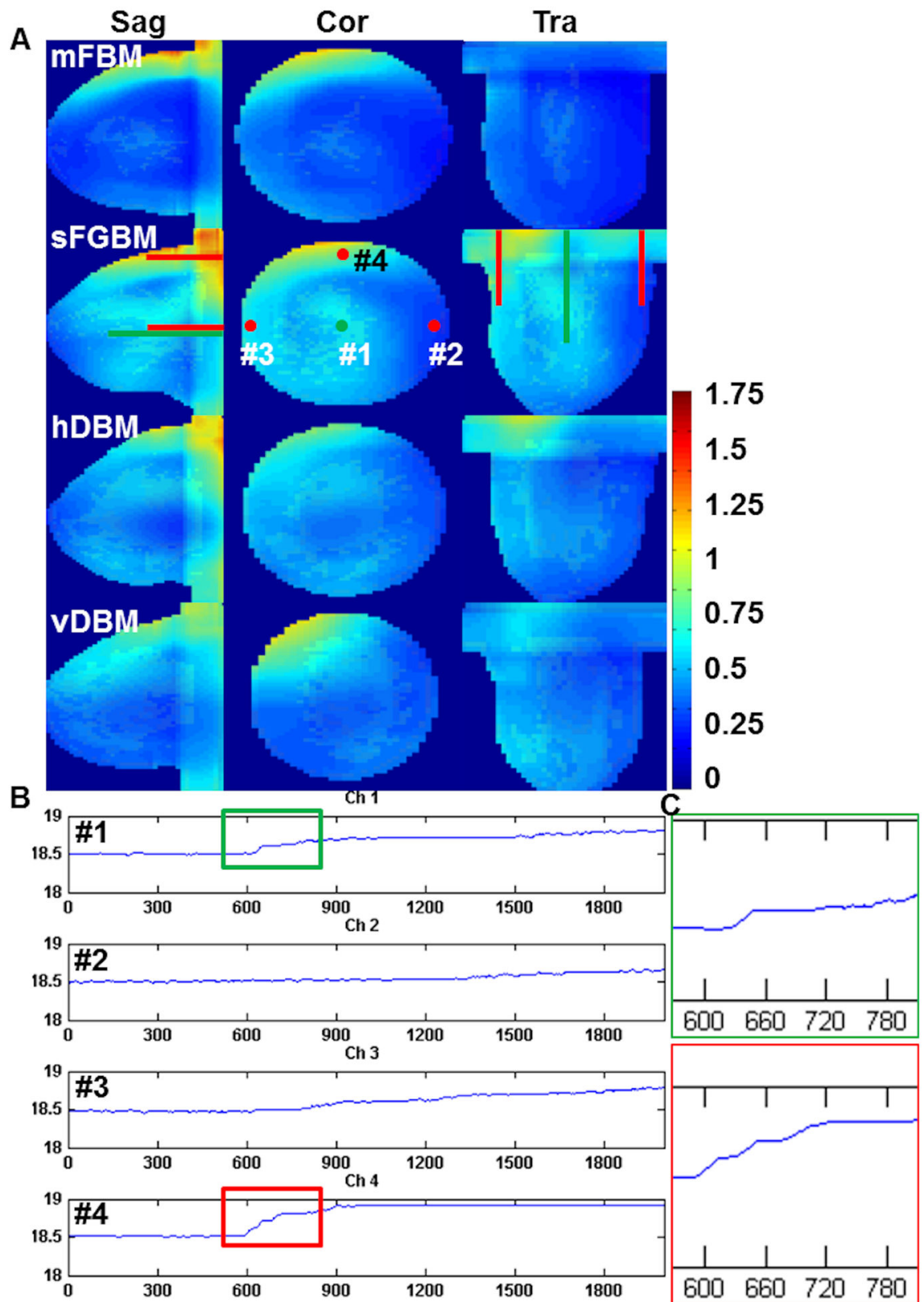

Figure 7.

(A) SAR (W/kg) for a mean $\mathrm{B}_{1}{ }^{+}$field of $2 \mu \mathrm{T}$ calculated in the four breast models. Peak SAR was measured close to the skin and chest wall region in all four models. The locations of the four thermal fiber optic probes used for the temperature measurements are shown as red and green bars. Three probes were inserted at the periphery of the phantom $(30 \mathrm{~mm}$ depth, red bars) and one probe was placed at the center of the phantom (50mm depth). Note, SAR map in four models were normalized to the highest calculated peak SAR (1.75 W/kg in sFGBM). (B) Temperature measurements at four locations in the Bphantom. (C) Zoomed-in view of 
the temperature rises measured from $\mathrm{Ch} 1$ and $\mathrm{Ch} 4$ during first RF heating. Note $\mathrm{RF}$ was applied at 600s and each scan was 2 min long and sequentially repeated for 5 times. 


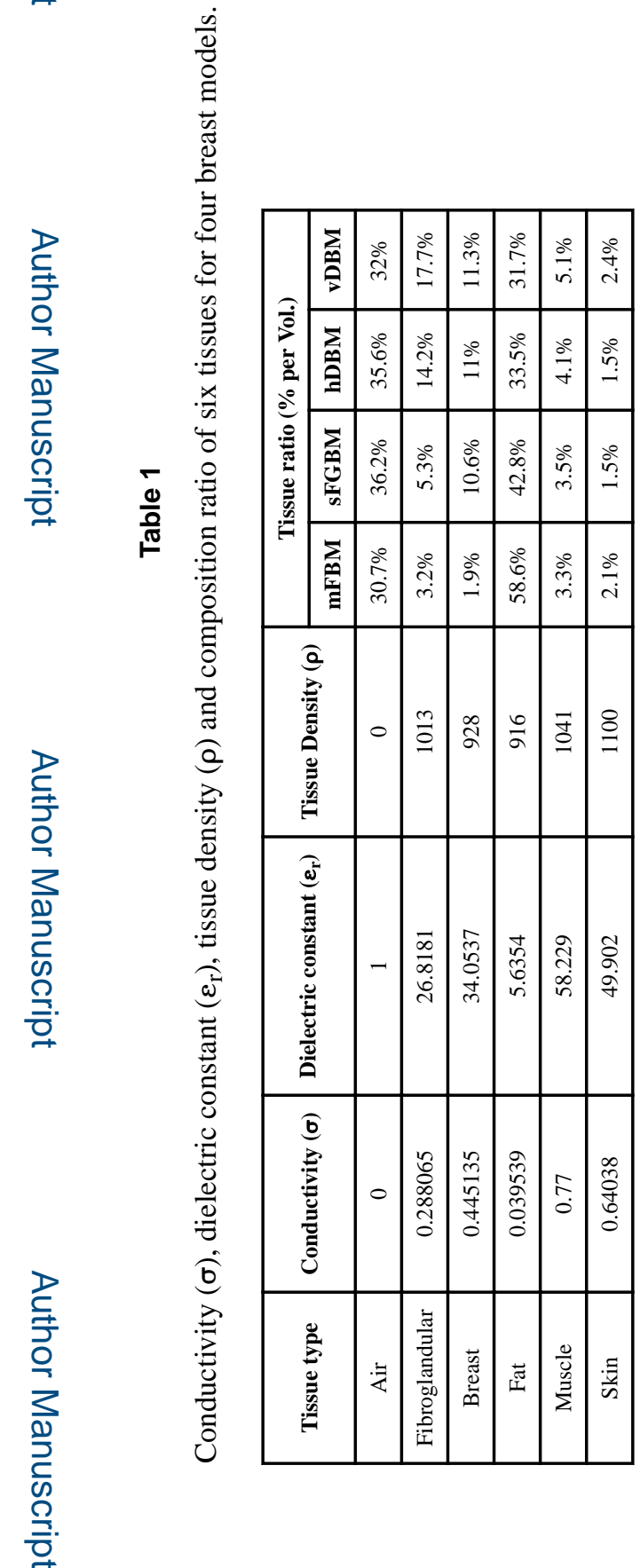

J Magn Reson. Author manuscript; available in PMC 2017 August 01. 


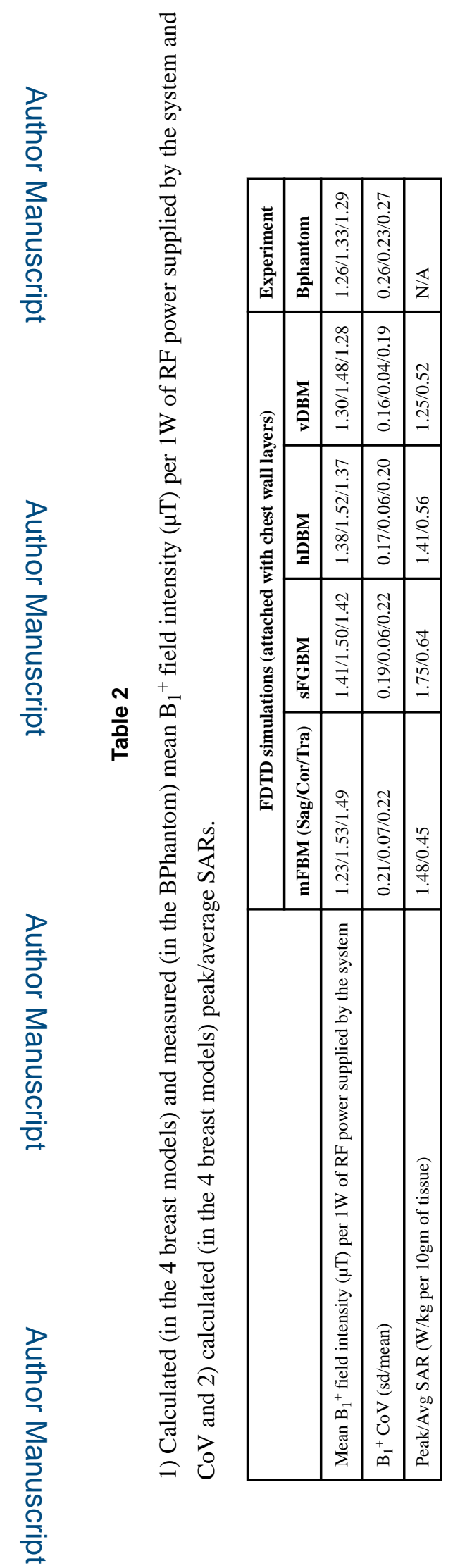

J Magn Reson. Author manuscript; available in PMC 2017 August 01. 


\section{Table 3}

Mean $\mathrm{B}_{1}{ }^{+}$field intensity $(\mu \mathrm{T})$ per $1 \mathrm{~W}$ of RF power supplied by the system, $\mathrm{CoV}$ and peak/average SARs comparison between sFGBM, Full body model, and without RF shield.

\begin{tabular}{|l|l|l|l|}
\hline & sFGBM & Full body model & without RF shield \\
\hline Mean $\mathrm{B}_{1}{ }^{+}$field intensity $(\mu \mathrm{T})$ per 1W of RF power supplied by the system & 1.53 & 1.26 & 0.82 \\
\hline $\mathrm{B}_{1}{ }^{+} \mathrm{CoV}(\mathrm{Sag} / \mathrm{Cor} / \mathrm{Tra})$ & $0.19 / 0.06 / 0.22$ & $0.2 / 0.09 / 0.22$ & $0.30 / 0.14 / 0.34$ \\
\hline Peak/Avg SAR (W/kg per 10gm of tissue) & $1.75 / 0.64$ & $2.34 / 0.64$ & $3.57 / 1.34$ \\
\hline
\end{tabular}

